\title{
A compact high-resolution X-ray ion mobility spectrometer
}

Cite as: Rev. Sci. Instrum. 87, 053120 (2016); https://doi.org/10.1063/1.4950866

Submitted: 11 March 2016 . Accepted: 05 May 2016 . Published Online: 23 May 2016

T. Reinecke, A. T. Kirk, A. Heptner, D. Niebuhr, S. Böttger, and S. Zimmermann

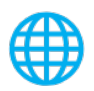

\section{ARTICLES YOU MAY BE INTERESTED IN}

Shutterless ion mobility spectrometer with fast pulsed electron source

Review of Scientific Instruments 88, 024102 (2017); https://doi.org/10.1063/1.4976021

Fast pulsed operation of a small non-radioactive electron source with continuous emission current control

Review of Scientific Instruments 86, 065102 (2015); https://doi.org/10.1063/1.4921707

Design and optimization of a corona discharge ionization source for ion mobility spectrometry

Review of Scientific Instruments 71, 2321 (2000); https://doi.org/10.1063/1.1150618

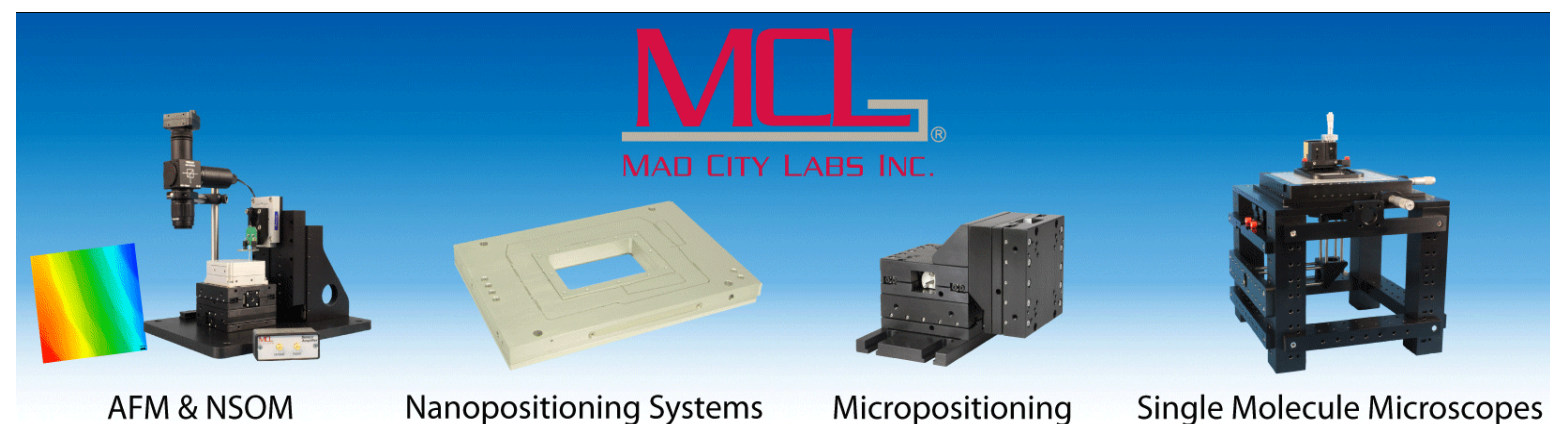




\title{
A compact high-resolution X-ray ion mobility spectrometer
}

\author{
T. Reinecke, A. T. Kirk, A. Heptner, D. Niebuhr, S. Böttger, and S. Zimmermann \\ Department of Sensors and Measurement Technology, Institute of Electrical Engineering and Measurement \\ Technology, Leibniz Universität Hannover, Appelstr. 9A, 30167 Hannover, Germany
}

(Received 11 March 2016; accepted 5 May 2016; published online 23 May 2016)

\begin{abstract}
For the ionization of gaseous samples, most ion mobility spectrometers employ radioactive ionization sources, e.g., containing ${ }^{63} \mathrm{Ni}$ or ${ }^{3} \mathrm{H}$. Besides legal restrictions, radioactive materials have the disadvantage of a constant radiation with predetermined intensity. In this work, we replaced the ${ }^{3} \mathrm{H}$ source of our previously described high-resolution ion mobility spectrometer with $75 \mathrm{~mm}$ drift tube length with a commercially available X-ray source. It is shown that the current configuration maintains the resolving power of $\mathrm{R}=100$ which was reported for the original setup containing a ${ }^{3} \mathrm{H}$ source. The main advantage of an X-ray source is that the intensity of the radiation can be adjusted by varying its operating parameters, i.e., filament current and acceleration voltage. At the expense of reduced resolving power, the sensitivity of the setup can be increased by increasing the activity of the source. Therefore, the performance of the setup can be adjusted to the specific requirements of any application. To investigate the relation between operating parameters of the X-Ray source and the performance of the ion mobility spectrometer, parametric studies of filament current and acceleration voltage are performed and the influence on resolving power, peak height, and noise is analyzed. Published by AIP Publishing. [http://dx.doi.org/10.1063/1.4950866]
\end{abstract}

\section{INTRODUCTION}

Ion mobility spectrometry (IMS) is a well-established technique for fast trace gas detection, offering limits of detection (LOD) in the low ppt $_{\mathrm{v}}$-range for measuring times of less than a second. The sensing principle of IMS is based on a separation of ions by their ion-specific mobility in a drift gas under the influence of an electric field. ${ }^{1}$ Due to its analytical performance and comparably compact realization, it is attractive for a wide range of applications. Commonly, IMS are used for security applications such as the detection of hazardous substances, ${ }^{2}$ drugs of abuse, ${ }^{3}$ or chemical warfare agents. ${ }^{4,5}$ However, today the field of other applications is constantly growing. IMS are used, e.g., in food industry ${ }^{6}$ or medical applications. ${ }^{7,8}$ However, as this technique is based on the separation of ions, every sample must be ionized prior to analysis. Different ionization sources offer different advantages and disadvantages, which must be considered when designing an instrument. A common method for the ionization of gaseous samples is radioactive $\beta^{-}$-radiators, often containing ${ }^{63} \mathrm{Ni}$ or ${ }^{3} \mathrm{H}$. One disadvantage of using radioactive materials in IMS is legal restrictions, complicating import, export, deployment in certain areas and disposal of such devices. Furthermore, the constant radiation with predefined intensity of radioactive ionization sources is disadvantageous, especially as it cannot be shut off. However, there are non-radioactive alternatives with variable intensity described in literature, e.g., using a UV photo ionization lamp, ${ }^{9,10}$ a corona discharge, ${ }^{11-15}$ a micro plasma, ${ }^{16}$ or nonradioactive electron emitters. ${ }^{17}$ Unfortunately, additionally to the reduced sensitivity when using UV ionization, the spectra gained from UV-photoionization and corona discharge differ from the spectra produced with a radioactive ionization sources, so they are not comparable. The main disadvantage of micro plasma and nonradioactive electron sources is that they are not widely commercially available.
Therefore, we investigate the performance of an X-ray source as an alternative ionization source to the tritium source of our previously described IMS. ${ }^{18}$ There are already some setups using X-ray IMS described in the literature. ${ }^{19,20}$ However, by now there is no comprehensive study on the impact of X-ray source working parameters on the performance of an IMS to be found.

\section{EXPERIMENTAL}

Fig. 1 depicts a schematic of the setup. It consists of a commercially available miniature X-ray source (XRT-502-Rh-0.6-125, Newton Scientific, Inc., USA), an ionization region between pulse and injection grid, a drift region, an aperture grid, and a Faraday detector. The X-ray source has a rhodium target with $0.6 \mu \mathrm{m}$ thickness and a focal spot size of $1 \mathrm{~mm} \times 2 \mathrm{~mm}$. The continuous power rating is $2 \mathrm{~W}$. The source is mounted axially in the position and orientation of the original ${ }^{3} \mathrm{H}$ source, as is beneficial for a simple realization of the mounting. Ions are injected by a field switching (FS) shutter, whose working principle is described in Ref. 21. It is based on switching the electrical field inside the ionization region on and off in order to control ion motion. The injection grid, dividing ionization region and drift region, is kept at a constant potential. To enable ion transmission from ionization region to drift region, a significantly higher potential than that of the injection grid is applied to the ion source. To stop ion transmission, the ionization region is kept field-free, which often requires a slightly lower potential on the ion source than on the injection grid in order to compensate field penetration through the injection grid. Here, an additional pulse grid was placed directly in front of the $\mathrm{X}$-ray source to serve as a replacement of the conductive surface of the metallic $\beta^{-}$-radiator. The advantages of the field switching shutter compared to the widely 


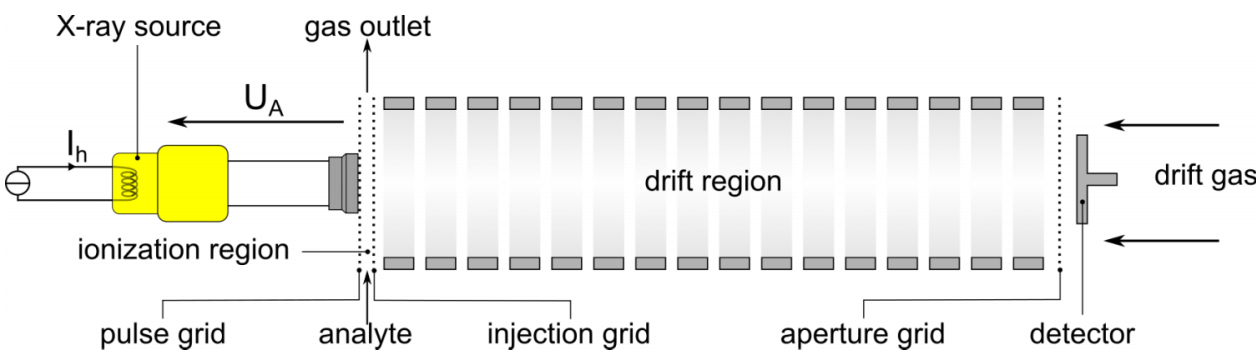

FIG. 1. Schematic of the X-ray-IMS.

used Bradbury-Nielsen (BN) shutter are easier manufacturing of the mechanical parts, improved mechanical stability, and reduced distortion of the drift field. The latter is the key for injecting short ion packages, which in turn is necessary for achieving high resolving power with compact drift tubes. The drift tube design used in this work is described in Ref. 18. Compared to that work, we use a tube with a reduced drift length of $75 \mathrm{~mm}$, as for example described in Ref. 22. A drift voltage of $4.9 \mathrm{kV}$ is applied via a resistive voltage divider over stainless steel ring electrodes separated by polyether ether ketone (PEEK) isolation rings, resulting in a homogeneous field inside the drift tube. The drift region is terminated with a faraday detector with aperture grid. The setup is operated at atmospheric pressure and a drift tube temperature of $23^{\circ} \mathrm{C}$. The IMS is purged with a gas flow of dry clean air at a flow rate of $250 \mathrm{ml} / \mathrm{min}$ and a dew point of $-85^{\circ} \mathrm{C}$. The sample gas flow is set to $11 \mathrm{ml} / \mathrm{min}$.

Fig. 2 shows a photo of the compact realization of our setup. The mounting for the X-ray source and the housing for the drift region are also comprised of polyether ether ketone (PEEK). To avoid analyte diffusion into the drift region, both the analyte gas inlet and the common gas outlet are localized at the ionization region.

\section{RESULTS AND DISCUSSION}

In first step, the influence of the operating parameters of the X-Ray source on the IMS-spectrum is investigated. As the X-ray source is mounted axially and the penetration depth of the radiation in air is much larger (few tens of $\mathrm{mm}$ depending on $\mathrm{U}_{\mathrm{A}}$ ) than the ionization region depth of the IMS, the neutral drift gas inside the drift region of the IMS is ionized continuously. This leads to an increasing offset current $\mathrm{I}_{\text {offset }}$ when increasing the activity of the $\mathrm{X}$-ray source. However, at first this is no drawback, as the offset can be simply

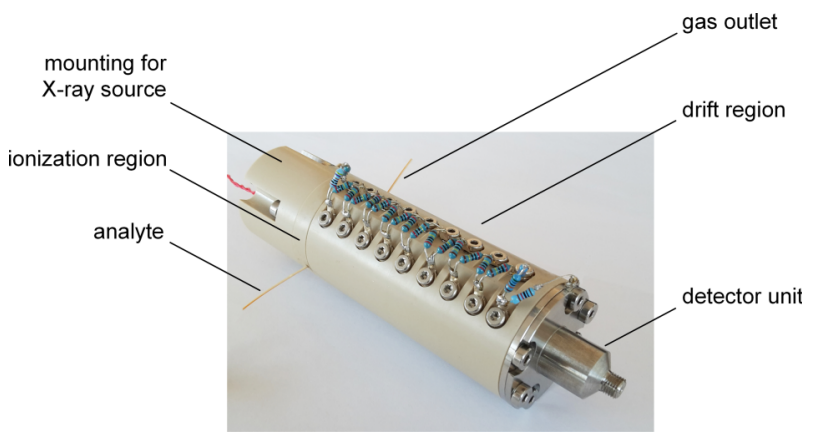

FIG. 2. Photo of the X-ray-IMS. subtracted from the spectrum for correction. Fig. 3 shows spectra of the positive reactant ion peak $\left(\mathrm{RIP}^{+}\right)$for arbitrary combinations of filament current $\mathrm{I}_{\mathrm{h}}$ and acceleration voltage $\mathrm{U}_{\mathrm{A}}$. Generally, increasing either $\mathrm{I}_{\mathrm{h}}$ or $\mathrm{U}_{\mathrm{A}}$ increases the intensity of the radiation, which results in a higher RIP $^{+}$intensity.

However, as shown in Fig. 4, it is not necessary to investigate these parameters on their own. It depicts the resolving power of the $\mathrm{RIP}^{+}$and the offset corrected $\mathrm{RIP}^{+}$-amplitude in dependency of the offset current, for different combinations of filament current and acceleration voltage. The resolving power, which is defined as the drift time of the corresponding peak divided by its full width at half maximum (FWHM), serves as a measure for the resolution of the setup, while the $\mathrm{RIP}^{+}$amplitude is used as a measure for the expected sensitivity of the setup. The RIP $^{+}$amplitude and analyte peak amplitude, and therefore sensitivity, should be proportional to each as long as the chemical ionization process is not influenced by the change of parameters.

It can be seen that the measurement data for three different filament currents with different acceleration voltages follow exactly the same function when using the offset current $\mathrm{I}_{\mathrm{offset}}$ as X-axis. This means that the same offset current produces the same IMS spectra, regardless of the combination of filament heating current and acceleration voltage that lead to this offset current. This is an extremely important observation, as filament heating current and acceleration voltage influence the $\mathrm{X}$-ray radiation in different ways. Changing the filament current only increases the intensity of the emitted X-radiation, whereas increasing the acceleration voltage also increases the intensity, but additionally shifts the low-wavelength cutoff to smaller wavelengths and therefore higher energies in the

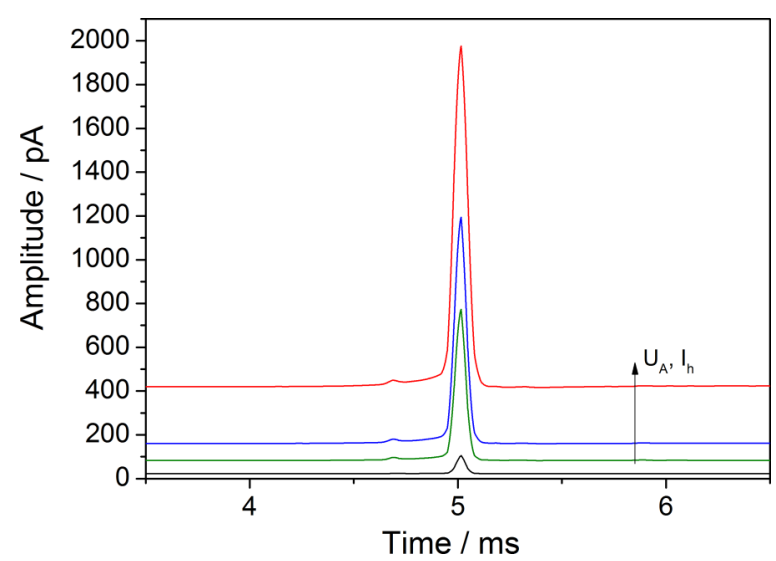

FIG. 3. RIP $^{+}$-spectra for different combinations of filament current and acceleration voltage. 


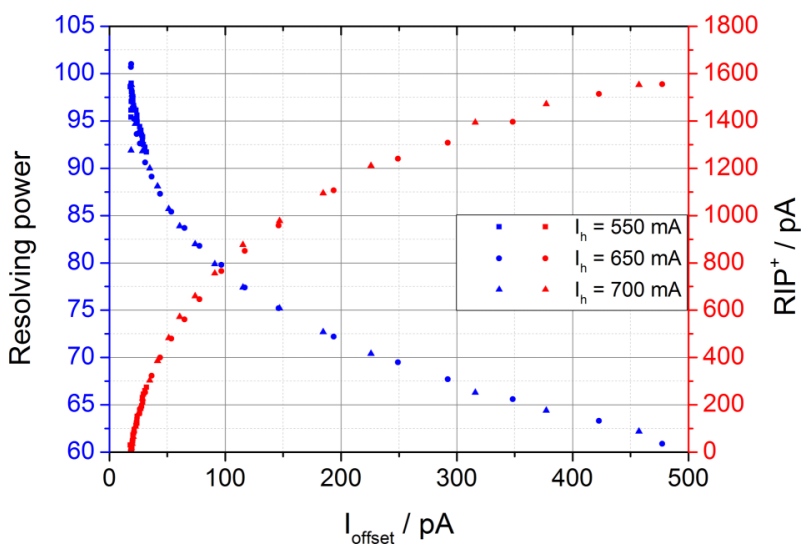

FIG. 4. Resolving power and $\mathrm{RIP}^{+}$-amplitude vs. offset current for different combinations of filament current and acceleration voltage.

emitted X-ray spectrum. Therefore, the offset current in the IMS spectrum is an ideal measure for the activity of the $\mathrm{X}$-ray source, as it reduces the parameters that need to be observed. For low offset currents, the resolving power of the $\mathrm{RIP}^{+}$is about $\mathrm{R}=100$, which is equal to the resolving power obtained using a ${ }^{3} \mathrm{H}$ source. Increasing the activity of the X-ray source leads to a decrease of the resolving power to $R=60$ at an offset current of $I_{\text {offset }}=475 \mathrm{pA}$ due to higher Coulomb repulsion. However, concurrently the amplitude of the $\mathrm{RIP}^{+}$is drastically increased from $7 \mathrm{pA}$ at $\mathrm{I}_{\text {offset }}=19 \mathrm{pA}-1560 \mathrm{pA}$ at $\mathrm{I}_{\text {offset }}=480 \mathrm{pA}$. Thus, it can be estimated that the sensitivity of the setup can be vastly improved choosing a higher activity of the X-ray source.

However, the true parameter of interest is not the sensitivity alone, but rather the limit of detection (LOD), which is defined as the peak intensity, which equals three times the noise. To make a statement about the limits of detection, the noise in the spectrum has to be considered additionally to the signal intensity. Fig. 5 shows the variance $\left(\sigma^{2}\right)$ of the noise after $2 \mathrm{~s}$ of averaging, plotted the offset current $\mathrm{I}_{\mathrm{Offset}}$. It can be seen that the variance of the noise increases linearly with increasing offset current. This observation can be explained with the phenomenon of Fano noise. ${ }^{23}$ Fano noise originates from the statistical nature of an X-ray ionization process and therefore the noise level is dependent on the number of ions produced. The variance of the Fano noise is expected to be

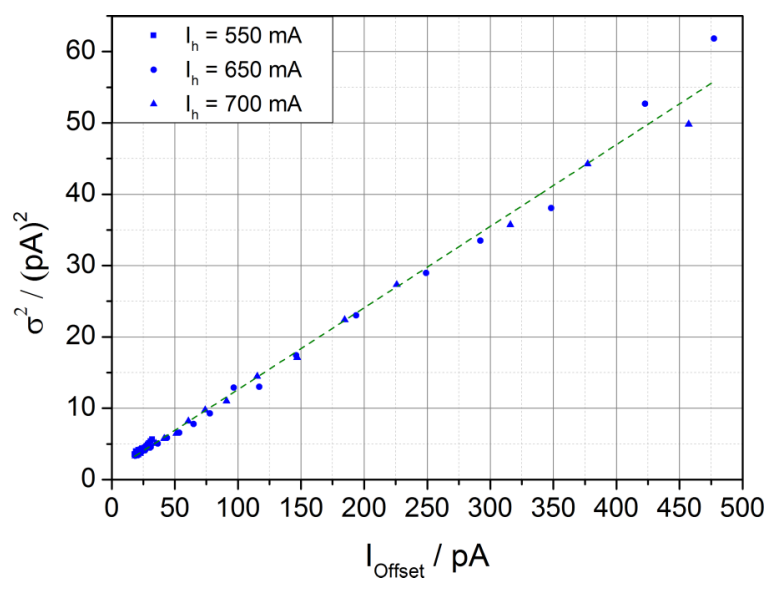

FIG. 5. Variance of noise in the spectrum vs. offset current.

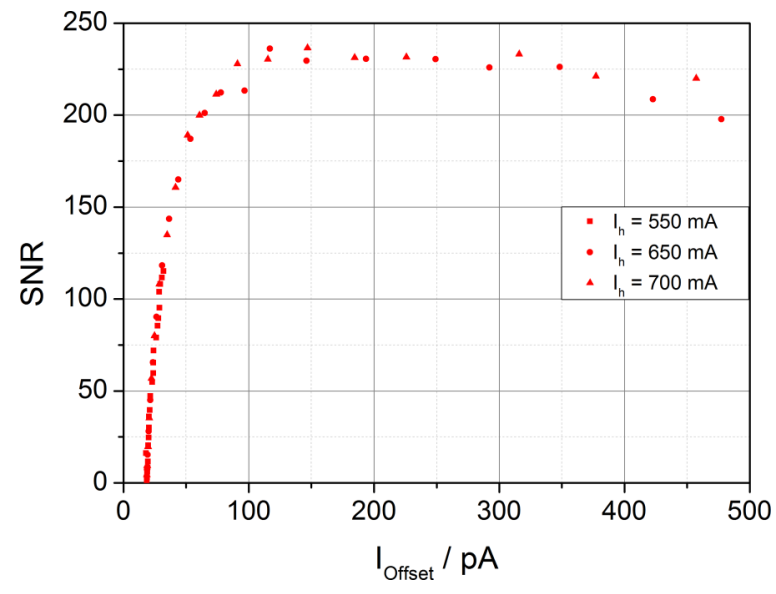

FIG. 6. Signal to noise ratio vs. offset current.

a linear function of the ion current, which agrees perfectly with the measurement shown in Fig. 5. Thus, we attribute the increase in measured noise current to the rising Fano noise.

As a measure for the limit of detection, the signal to

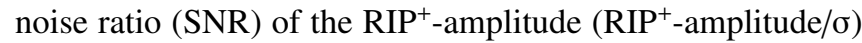
is investigated as shown in Fig. 6. It can be seen that the highest SNR (i.e., the best expected limit of detection) of about $\mathrm{SNR}=240$ is already reached at an offset current in the range of $\mathrm{I}_{\text {offset }}=100 \mathrm{pA}-150 \mathrm{pA}$, although in this range the $\mathrm{RIP}^{+}$-amplitude is still massively increasing with increasing offset current, as shown in Fig. 4. However, as the increasing noise limits the maximum SNR, better limits of detection are achieved at intermediate amplitudes. Therefore, the best overall performance is achieved with an X-ray source activity at the lower limit of the given offset current range that maximizes SNR, i.e., $\mathrm{I}_{\text {offset }}=100 \mathrm{pA}$, as it combines the maximum SNR with higher resolving power than that achieved using higher offset currents.

Having determined the optimum activity of the $\mathrm{X}$-ray source, we performed measurements on two different chemicals in clean dry air. Acetone was characterized in positive ion mode, while methyl salicylate was characterized in the negative ion mode, in which all voltage polarities are inverted. In the positive mode, the positive reactant ion peak $\left(\mathrm{RIP}^{+}\right)$, an acetone monomer and an acetone dimer are observed as shown in Fig. 7(a)). This spectrum is equivalent to that observed using a ${ }^{3} \mathrm{H}$ source. In the negative mode spectrum shown in Fig. 7(b), a double negative reactant ion peak (RIP ${ }^{-}$) and a single methyl salicylate peak appear. Again, this mirrors the spectrum observed with the ${ }^{3} \mathrm{H}$ source.

For both substances, limits of detection were determined based on the measured noise and the measured linear amplitude increase of the product ion peaks at low concentrations. The limit of detection was defined as three times the standard deviation of the noise. For a measuring time of $2 \mathrm{~s}$, the achieved limits of detection are $86 \mathrm{ppt}_{\mathrm{v}}$ for acetone using the monomer and $420 \mathrm{ppt}_{\mathrm{v}}$ for methyl salicylate. The limits of detection achieved with a similar setup employing a ${ }^{3} \mathrm{H}$ source under similar conditions are $57 \mathrm{ppt}_{\mathrm{v}}$ for acetone and $127 \mathrm{ppt}_{\mathrm{v}}$ for methyl salicylate. However, these values are not completely comparable, as implementation of the X-ray source mounting leads to a change of the gas outlet position and the geometry of the ionization region. 


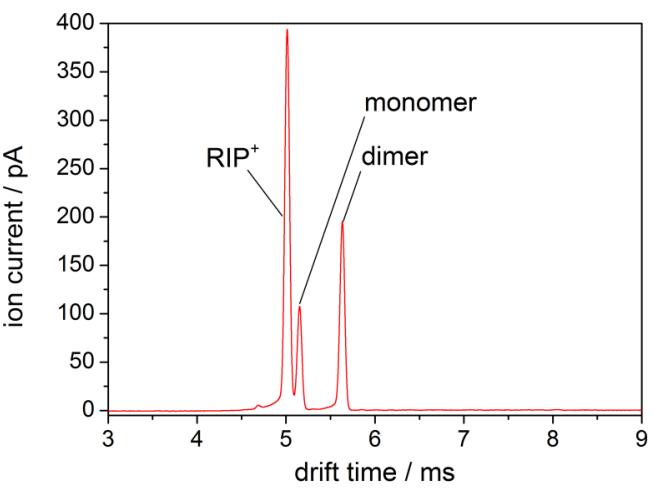

(a)

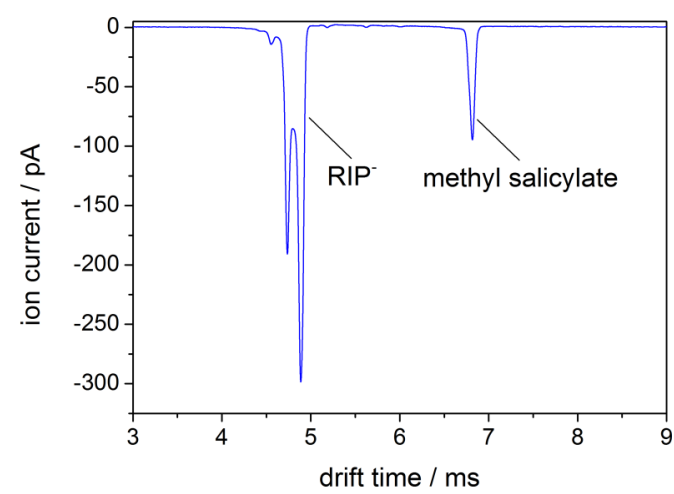

(b)

FIG. 7. Positive IMS-spectrum of acetone in clean dry air (a) and negative IMS-spectrum of methyl salicylate in clean dry air (b).

\section{CONCLUSION}

In this work, we present the realization and characterization of an X-ray IMS. Therefore, the ${ }^{3} \mathrm{H}$-source of our previously described compact high resolution IMS was replaced by a commercially available X-ray source. The realized setup maintains the resolving power of $\mathrm{R}=100$ at a drift length of $75 \mathrm{~mm}$. A systematic investigation of the operating parameters of the X-ray source showed that the signal intensity can be increased by increasing either the filament current or the acceleration voltage. Furthermore, the offset current in the spectrum, which is created due to the axial orientation of the $\mathrm{x}$-ray source, proved to be an ideal measure for the activity of the source, as it could be shown that every combination of filament current and acceleration voltage with identical offset current results in an identical IMS-spectrum. However, increasing the offset current also leads to increasing noise in the spectrum. As the variance of the noise is proportional to the offset current, the increasing noise was attributed to Fano noise, i.e., the statistical behavior of the X-ray ionization process. Therefore, the maximum signal to noise ratio is limited as at some point the increase in signal amplitude is lower than the increase in noise. Measurements of acetone in positive mode and methyl salicylate in negative mode using an optimized $\mathrm{X}$-ray source activity showed very good detection limits in the low $\mathrm{ppt}_{\mathrm{v}}$-range.

${ }^{1}$ G. A. Eiceman, Z. Karpas, and H. H. Hill, Ion Mobility Spectrometry, 3rd ed. (CRC Press, Boca Raton, 2013).

${ }^{2}$ G. A. Eiceman, A. P. Snyder, and D. A. Blyth, "Monitoring of airborne organic vapors using ion mobility spectrometry," Int. J. Environ. Anal. Chem. 38(3), 415-425 (1990).

${ }^{3}$ A. B. Kanu and H. H. Hill, "Identity confirmation of drugs and explosives in ion mobility spectrometry using a secondary drift gas," Talanta 73(4), 692-699 (2007).

${ }^{4}$ R. G. Ewing, D. A. Atkinson, G. A. Eiceman, and G. J. Ewing, "A critical review of ion mobility spectrometry for the detection of explosives and explosive related compounds," Talanta 54(3), 515-529 (2001).

${ }^{5}$ C. Wu, W. E. Steiner, P. S. Tornatore, L. M. Matz, W. F. Siems, D. A. Atkinson, and H. H. Hill, "Construction and characterization of a high-flow, high-resolution ion mobility spectrometer for detection of explosives after personnel portal sampling," Talanta 57(1), 123-134 (2002).

${ }^{6}$ W. Vautz, D. Zimmermann, M. Hartmann, J. I. Baumbach, J. Nolte, and J. Jung, "Ion mobility spectrometry for food quality and safety," Food Addit. Contam. 23(11), 1064-1073 (2006).
${ }^{7}$ M. Jünger, W. Vautz, M. Kuhns, L. Hofmann, S. Ulbricht, J. I. Baumbach, M. Quintel, and T. Perl, "Ion mobility spectrometry for microbial volatile organic compounds: A new identification tool for human pathogenic bacteria," Appl. Microbiol. Biotechnol. 93(6), 2603-2614 (2012).

${ }^{8}$ W. Vautz, J. I. Baumbach, M. Westhoff, K. Züchner, E. T. H. Carstens, and T. Perl, "Breath sampling control for medical application," Int. J. Ion Mobility Spectrom. 13(1), 41-46 (2010).

9J. I. Baumbach, S. Sielemann, Z. Xie, and H. Schmidt, "Detection of the gasoline components methyl tert -butyl ether, benzene, toluene, and $\mathrm{m}$ xylene using ion mobility spectrometers with a radioactive and UV ionization source," Anal. Chem. 75(6), 1483-1490 (2003).

${ }^{10}$ St. Sielemann, J. I. Baumbach, H. Schmidt, and P. Pilzecker, "Detection of alcohols using UV-ion mobility spetrometers," Anal. Chim. Acta 431(2), 293-301 (2001).

${ }^{11} \mathrm{H}$. Borsdorf, "Determination of n-alkanes and branched chain alkanes by corona discharge ion mobility spectrometry,” Int. J. Ion Mobility Spectrom. 2(1), 9-14 (1999).

${ }^{12}$ H. Borsdorf, H. Schelhorn, J. Flachowsky, H.-R. Döring, and J. Stach, "Corona discharge ion mobility spectrometry of aliphatic and aromatic hydrocarbons," Anal. Chim. Acta 403(1-2), 235-242 (2000).

${ }^{13}$ C. L. Crawford and H. H. Hill, "Comparison of reactant and analyte ions for ${ }^{63} \mathrm{Nickel}$, corona discharge, and secondary electrospray ionization sources with ion mobility-mass spectrometry," Talanta 107, 225-232 (2013).

${ }^{14}$ G. A. Eiceman, J. H. Kremer, A. P. Snyder, and J. K. Tofferi, "Quantitative assessment of a corona discharge ion source in atmospheric pressure ionization-mass spectrometry for ambient air monitoring," Int. J. Environ. Anal. Chem. 33(3-4), 161-183 (1988).

${ }^{15} \mathrm{M}$. Tabrizchi and A. Abedi, "Negative corona discharge ionization source for ion mobility spectrometry," Int. J. Ion Mobility Spectrom. 5, 51-54 (2002).

${ }^{16}$ W. Vautz, A. Michels, and J. Franzke, "Micro-plasma: A novel ionisation source for ion mobility spectrometry," Anal. Bioanal. Chem. 391(7), 2609-2615 (2008).

${ }^{17}$ P. Cochems, M. Runge, and S. Zimmermann, "A current controlled miniaturized non-radioactive electron emitter for atmospheric pressure chemical ionization based on thermionic emission," Sens. Actuators, A 206, 165-170 (2014).

${ }^{18}$ A. T. Kirk, M. Allers, P. Cochems, J. Langejuergen, and S. Zimmermann, "A compact high resolution ion mobility spectrometer for fast trace gas analysis," The Analyst 138(18), 5200-5207 (2013).

${ }^{19}$ V. S. Pershenkov, A. D. Tremasov, V. V. Belyakov, A. U. Razvalyaev, and V. S. Mochkin, "X-ray ion mobility spectrometer," Microelectron. Reliab. 46(2-4), 641-644 (2006).

${ }^{20}$ H.-R. Döring, "Ion mobility spectrometer with mechanically stabilized vacuum-tight X-ray window,” U.S. patent US6740874 (B2) (25 May 2004).

${ }^{21}$ A. T. Kirk and S. Zimmermann, "Bradbury-Nielsen vs. Field switching shutters for high resolution drift tube ion mobility spectrometers," Int. J. Ion Mobility Spectrom. 17(3-4), 131-137 (2014).

${ }^{22}$ A. T. Kirk and S. Zimmermann, "An analytical model for the optimum drift voltage of drift tube ion mobility spectrometers with respect to resolving power and detection limits,' Int. J. Ion Mobility Spectrom. 18(3-4), 129-135 (2015).

${ }^{23}$ U. Fano, "Ionization yield of radiations. II. The fluctuations of the number of ions," Phys. Rev. 72(1), 26-29 (1947). 